\title{
Data Collection and the Questionnaires for the Effective Use of Biobank for Metabolic Disorders
}

\author{
Hidekatsu Yanai ${ }^{a}$, b, c , Yukari Takano ${ }^{\mathrm{b}}$, Hiroki Adachi ${ }^{\mathrm{a}}$, Akiko Kawaguchia ${ }^{\mathrm{a}}$, Mariko Hakoshima ${ }^{\mathrm{a}}$, \\ Yoko Waragai $^{\text {a }}$, Tadanao Harigae ${ }^{a}$, Hidetaka Hamasaki ${ }^{a}$, Hisayuki Katsuyama ${ }^{\text {a }}$, \\ Tomoko Kaga $^{\mathrm{b}}$, Akahito Sako ${ }^{\mathrm{a}, \mathrm{b}}$
}

Recently, the number of excellent original articles about genetics such as global transcript profiling and genetic ancestry has been increasing, due to that "Journal of Endocrinology and Metabolism (JEM)" became widely known [1-3]. I wish further development of JEM as Editor-in-Chief. Here, we will introduce Biobank which may induce further development of studies on genetics, endocrinology and metabolism, and will also show the questionnaires for the effective use of Biobank for metabolic disorders.

The idea for Biobank began in 1998 when UK government spending review gave the Medical Research Council additional funds to set up "nationally available DNA collections" [4]. We started to set up the Biobank for Metabolic Disorders in the National Center for Global Health and Medicine Kohnodai Hospital with financial support from the Biobank Fund of National Center for Global Health and Medicine. The Biobank project is a prospective cohort study to collect baseline data and blood samples from individuals who will be followed up, to find the correlation of disease with genetic, environmental exposure, and baseline data [4]. When disease developed, prospectively collected samples linked to baseline data and baseline data themselves can be used to look back for evidence of exposure [4].

Genetic and environmental factors are associated with development of metabolic disorders. Genetic factors may modify the influences of environmental factors, and environmental factors including lifestyle-related behaviors may also enhance or reduce the effects of genetic factors, which may be further modified by gender and aging [5]. To understand the pathogenesis of metabolic disorders, Biobank may be a useful tool.

In our Biobank, informed consent (IC) is obtained after the explanation of Biobank. After IC was obtained, we collected the baseline data using the questionnaires about diabetes-related and other health-related factors, lifestyle including eating behaviors [6]. At the first visit after the obtainment of IC, DNA and serum are obtained from patients. Serum and

Manuscript accepted for publication February 15, 2017

aDepartment of Internal Medicine, National Center for Global Health and Medicine Kohnodai Hospital, Chiba, Japan

${ }^{\mathrm{b} C l i n i c a l}$ Research and Trial Center, National Center for Global Health and Medicine Kohnodai Hospital, Chiba, Japan

${ }^{\mathrm{c}}$ Corresponding Author: Hidekatsu Yanai, Department of Internal Medicine, National Center for Global Health and Medicine Kohnodai Hospital, 1-7-1 Kohnodai, Ichikawa, Chiba 272-0034, Japan.

Email: dyanai@hospk.ncgm.go.jp

doi: https://doi.org/10.14740/jem400w medical records including laboratory data, body weight, blood pressure and medication are obtained at every visit.

We believe that the obtainment of valid and abundant baseline data is essential to make the useful Biobank for metabolic disorders.

Here, we will introduce our questionnaires to obtain diabetes-related data (Table 1), other health-related data (Table 2 ) and the data about lifestyle (Table 3), which may help you study the metabolic disorders. We will be happy that our questionnaires lead your study to success.

\section{Conflicts of Interest}

The authors declare that they have no conflicts of interest concerning this article.

\section{Funding Sources}

This work was funded by a research grant from the Biobank Fund of National Center for Global Health and Medicine.

\section{References}

1. Doumatey AP, Xu H, Huang H, Trivedi NS, Lei L, Elkahloun A, Adeyemo A, et al. Global Gene Expression Profiling in Omental Adipose Tissue of Morbidly Obese Diabetic African Americans. J Endocrinol Metab. 2015;5(3):199-210.

2. Luk HM. Clinical and Genetic Study of Pseudohypoparathyroidism Type $1 \mathrm{~b}$ in Hong Kong Chinese. J Endocrinol Metab. 2016;6(2):64-70.

3. de Queiroz EM, Barbosa PO, Candido AP, Castro IM, Machado-Coelho GLL, Leite TM, Pereira RW, de Freitas RN. Genetic Ancestry Is Associated With Systolic Blood Pressure and Glucose in Brazilian Children and Adolescents. J Endocrinol Metab. 2016;6(6):167-171.

4. Barbour V. UK Biobank: a project in search of a protocol? Lancet. 2003;361(9370):1734-1738.

5. Yanai H. The Metabolic Disorder and Metabolic Disorder-Related Diseases Model. J Endocrinol Metab. 2016;6(6):165-166.

6. Yoshimatsu H. [Behavioral therapy for obesity]. Nihon Rinsho. 2009;67(2):373-383. 


\section{Table 1. Diabetes-Related Data}

1. Basic data

Age, year of birth, sex, dominant arm (right or left)

2. Anthropometric data

Body height $(\mathrm{cm})$, body weight $(\mathrm{kg})$, abdominal circumference $(\mathrm{cm})$, systolic and diastolic blood pressure $(\mathrm{mm} \mathrm{Hg})$, heart rate $($ beats $/ \mathrm{min})$, grip strength $(\mathrm{kg})$

3. The questionnaires about body weight

Did your body weight change?

Yes or no

If you answered "yes"

A. How did your body weight change?

4. The questionnaires about diabetes

Were diabetes or hyperglycemia pointed out to you?

Yes or no

If you answered "yes"

A. When were you told that your plasma glucose was high? (day/month/ year) or at the age of ___ years old

B. Why did you know that your plasma glucose was high?

You (gained or lost) __ $\mathrm{kg}$ in ___ (weeks, months, years)

5. The questionnaires about symptoms

Do you have the following symptoms?

- Thirsty

- General fatigue

- Chest pain or chest discomfort during exercise

- Drink plenty of water

- Get tired easily

- Palpitation or short of breath

- Frequent urination

- Edema of face and/or limbs

- Amenorrhea

- Increased or decreased appetite

- Numbness in toe and/or planta

- Erectile dysfunction

- Foot pain

a) Medical checkup

b) When you visited hospital because you have symptoms of diabetes.

c) When you visited hospital because you have other diseases. What are other diseases?

d) Other reasons

6. The questionnaires about the treatment for diabetes

Did or do you receive the treatment for diabetes?

Yes or no

If you answered "yes"

A. Did or do you take oral anti-diabetic drugs?

The date of start and cessation of the drugs, and the name of drugs which you used

B. Were or are you treated by insulin

The date of start and cessation of insulin, and the name of insulin which you used

C. Only exercise therapy

a) What kind of exercise?

b) How long do you exercise?

c) How often do you exercise?

- Scratches are difficult to heal

- Cramp

- Skin ulcer

- Weight gain or loss

- Athlete's foot

- Depression

- Decreased visual acuity

- Sensitivity to cold or cool limbs

- Insomnia or sleep disorder

- Taste disorder

- Constipation or diarrhea

- Other symptoms

D. Only diet therapy

a) How is your diet therapy?

b) How much calorie do you take a day?

E. Did you receive nutritional education?

Yes or no

If you answered "yes"

a) salt ___ g a day, protein __ g day, potassium _ _ mg a day

F. Did you experience the admission to hospital to receive the treatment or education for diabetes?

Yes or no

If you answered "yes"

a) The name of hospital, the date of admission

7. The questionnaires about familial history of diabetes

Do you have the relatives with diabetes?

Yes or no

If you answered "yes"

A. Who

grandfather, grandmother, father, mother, brother, sister

B. The treatment for diabetes

Only diet or exercise therapy, oral anti-diabetic drugs, insulin

C. Diabetic complication

Hemodialysis, blindness, gangrene or amputation of lower limb, cerebral infarction, angina or myocardial infarction, cerebral hemorrhage, other complications 


\section{Table 2. Other Health-Related Data}

1. The questionnaires about the medical checkup Regular medical checkup, the medical checkup for cancer

2. For females, the questionnaires about menstruation, pregnancy and delivery

A. Do you have menstruation?

Yes or no

B. Did you experience pregnancy and/or delivery?

If you answered "yes"

Did you have medical problems during pregnancy?

3. The questionnaires about allergy

A. Do you have allergy?

Yes or no

If you answered "yes"

B. What are you allergic to?

- Milk

- Soy

- Egg

- Pollen

- Mackerel

- Mite

C. Do you have atopic dermatitis?

Yes or no

D. Do you have drug allergy?

Yes or no

a) pregnancy-induced hypertension

b) hyperglycemia and/or urinary glucose during pregnancy

c) other medical problems during pregnancy

C. How much was your child's body weight at birth?

4. The questionnaires about the current and past treatment for other diseases

- Hypertension

- Hyperuricemia and/or gout

- Pollinosis

- Arrhythmia

- Renal diseases

- Depression

- Angina

- Bronchial asthma

- Myocardial infarction

- Soba

- $\mathrm{Crab}$

- Wheat

- Shrimp

- Other foods

If you answered "yes"

a) The name of drug

b) Symptoms due to drug allergy

- Tuberculosis

- Adrenal insufficiency

- Hyperlipidemia

- Cerebral infarction

- Rheumatoid arthritis

- Chronic hepatitis

- Peripheral artery diseases

- Malignancy

- Other diseases

5. The questionnaires about the operation

A. Did you receive the operation?

Yes or no

a) The disease treated by operation, and operative procedure If you answered "yes"

b) The date of the operation

6. The questionnaires about familial history of other diseases

Hypertension, angina, myocardial infarction, malignancy, hyperlipidemia, hyperuricemia and/or gout, renal diseases, cerebral infarction and other diseases 
Table 3. The Data About Lifestyle

1. Diet

A. Do you have the following eating habits?

- Cook by yourself

- Family cook for you

- Eating out is often

- Chew well when eating.

- Eating quickly

- Snack a lot

- Like sweets

- Prefer fish to meat

- Eating fried foods is often

2. Smoking

Do you smoke?

A. non-smoker

B. ex-smoker

C. current smoker

3. Alcohol drink

Do you drink alcohol?
A. No
B. used to drink
C. Yes
If you answered $\mathrm{B}$ or $\mathrm{C}$

a) What's kind of alcohol did or do you drink?

4. Exercise

A. Do you take moderate exercise except for walking? No,

Yes, everyday; __ times a week; __ times a month
What kind of exercise?

How long? minutes

5. Work

What is your work?

- Light work such as desk work, housewife, student, or un-employee

- Moderate work such as standing for a long time to do the job

6. Sleep and mental health

A. How long do you sleep

Bedtime

Wake-up time _

7. Body weight

A. How much did you weigh when you were the heaviest in the past?

If you are women, exclude those when you were pregnancy. $\mathrm{kg}$ at the age of years old
- Midnight snack is often

- Irregular meal time

- Skipping breakfast is often

- A late dinner What time do you eat dinner?

- Have a habit of drinking green tea How many cups of green tea do you drink a day?

- Have a habit of drinking coffee How many cups of coffee do you drink a day?

If you answered $\mathrm{B}$ or $\mathrm{C}$

How many cigarettes did you smoke a day? cigarettes a day for years

sake, wine, beer, distilled spirits, others

b) How much did or do you drink a day? $\mathrm{mL}$,__ grasses a day

c) How often did or do you drink? __ days a week, __ days a month

B. Do you walk for 15 minutes or more? No,

Yes, everyday; __ times a week; times a

month

How long? minutes

- Heavy work such as heavy labor

- Shift work

- Taxi driver

- Sales job

Sleeping hours: __ hours and __ minutes

B. Feeling stressed is often Yes or no

B. How much did you weigh when you were 20 years old? $\mathrm{kg}$ 